\title{
The effects of Kv1.3 and IKCa1 channel inhibition on cytokine production and calcium influx of $T$ lymphocytes in rheumatoid arthritis and ankylosing spondylitis
}

\author{
Gergely Toldi $^{1,2} \cdot$ Luis Munoz $^{3} \cdot$ Martin Herrmann $^{3} \cdot$ Georg Schett ${ }^{3}$. \\ Attila Balog 4
}

Published online: 18 August 2015

(C) Springer Science+Business Media New York 2015

\begin{abstract}
Kv} 1.3$ and IKCa1 lymphocyte potassium channels have been implicated as important targets of selective immunomodulation. We compared the alterations in cytokine production upon selective inhibition of Kv1.3 or IKCa 1 channels (by MGTX and TRAM, respectively) in healthy donors (HD), RA and AS patients. We also determined calcium influx kinetics and its sensitivity to Kv1.3 and IKCa1 channel inhibition following PHA activation in CD4, Th1, Th2 and CD8 cells as well as monocytes. The application of TRAM resulted in a lower production of TNF-a and IL1-RA in all three study groups. Inhibition by TRAM had contrary effects on the production of IL-1b and IL-5: While their production was increased by PBMCs of RA patients, this effect was not observed in HD and AS PBMCs. While treatment with MGTX resulted in a similar decrease in calcium influx in the CD4 and Th2 subsets across all study groups, TRAM treatment had opposite effects on RA and HD samples: It decreased calcium influx in the Th2 and CD8 subsets in RA, while only Th1 cells were affected in HDs. The effects of IKCa1 channel inhibition are controversial in samples of RA and AS patients, since it shifts the inflammatory balance into the pro-inflammatory direction.
\end{abstract}

Gergely Toldi

toldigergely@yahoo.com

1 First Department of Pediatrics, Semmelweis University, Bókay u. 53, Budapest 1083, Hungary

2 MTA-SE, Pediatrics and Nephrology Research Group, Budapest, Hungary

3 Department of Internal Medicine 3, University of ErlangenNürnberg, Erlangen, Germany

4 Department of Rheumatology, University of Szeged, Szeged, Hungary
Keywords AS - Calcium influx - Cytokine - IKCa1 . $\mathrm{Kv} 1.3 \cdot \mathrm{RA}$

\section{Introduction}

Voltage-sensitive Kv1.3 and calcium-dependent IKCa1 lymphocyte potassium channels have been implicated as important targets of selective immunomodulation in autoimmune disorders. The transient increase in the cytoplasmic-free calcium level, which is a prerequisite of the downstream events of lymphocyte activation, is maintained by the function of potassium channels. They conserve the electrochemical potential gradient via the efflux of potassium from cytoplasm [1]. The relationship between the influx of calcium through the cell membrane and the efflux of potassium makes proliferation and activation of lymphocytes sensitive to pharmacological inhibition of Kv1.3 and IKCa1 channels and provides a potential opportunity for targeted intervention.

Earlier studies reported that disease-associated autoreactive $\mathrm{T}$ cells from patients with rheumatoid arthritis (RA) are mainly effector memory $\mathrm{T}$ cells (TEM cells) with elevated Kv1.3 channel expression. In contrast, T cells with other antigen specificities from these patients are predominantly naive or central memory $\mathrm{T}$ cells (TCM cells), expressing low levels of $\mathrm{Kv} 1.3$ that upregulate IKCa1 channels upon activation. Therefore, selective inhibition of Kv1.3 channels was implicated to specifically suppress disease-associated, autoantigen-specific TEM cells, while sparing other $\mathrm{T}$ cell subsets. Indeed, Kv1.3 inhibitors ameliorated pristane-induced arthritis in rats without systemic toxic effects [2]. Previous data indicated that specific inhibition of the Kv1.3 channel indeed acts differentially 
on calcium influx kinetics in major peripheral blood lymphocyte subsets of RA patients. Unlike in healthy individuals, Th2, and in particular, CD8 cells are inhibited more dominantly than Th1 and CD4 cells. However, the inhibitory effect does not seem to be as specific as reported in case of autoreactive T cells, since Th 2 cells are affected as well [3].

Limited data are available on the effects of lymphocyte potassium channel inhibition on cytokine production and its relation to the regulation of calcium influx, especially in cytokine-producing Th1 and Th2 subsets. Over the recent years, targeted therapies have been developed to prevent the harmful effect of certain cytokines, such as TNF-a, IL-6 or IL-1b. The success of these biological therapies demonstrates the need to further study interventional methods influencing cytokine production.

In this study, we aimed to compare the alterations in cytokine production patterns caused by selective inhibition of the Kv1.3 or IKCa1 channels in healthy donors, as well as in patients with RA or ankylosing spondylitis (AS). To evaluate the mechanisms that potentially contribute to these alterations, we also investigated calcium influx kinetics in peripheral blood mononuclear cells and the effects of inhibitors of $\mathrm{Kv} 1.3$ and IKCa1 potassium channels.

\section{Materials and methods}

\section{Patients}

Peripheral blood samples were collected from 15 RA patients, 10 AS patients and 8 healthy donors (HD). Clinical data of participants are summarized in Table 1 . HD had a negative history of rheumatic symptoms and negative status upon detailed physical and laboratory examination.
Written informed consent was obtained from all subjects, and our study was reviewed and approved by the ethics committee of the institution. The study adhered to the tenets of the most recent revision of the Declaration of Helsinki.

\section{Cytokine levels}

For the measurement of cytokines from supernatants, $5 \times 10^{5}$ PBMCs were cultured in RPMI medium supplemented with $\mathrm{CaCl}_{2}$ (calcium concentration: $2 \mathrm{mM}$ ) over $48 \mathrm{~h}$ in the presence of $160 \mathrm{nM}$ PMA and in the presence or absence of $4 \mathrm{nM}$ margatoxin (MGTX) or $240 \mathrm{nM}$ triarylmethane-34 (TRAM). Then, supernatants were frozen at $-80 \mathrm{C}$ until analysis. Cytokine concentrations (IL-1b, IL1-RA, IL-2, IL-4, IL-5, IL-6, IL-10, IL-12p70, IL-13, IL$17 \mathrm{~A} / \mathrm{F}, \mathrm{IFN}-\mathrm{g}, \mathrm{TNF}-\mathrm{a})$ were determined using the eBioscience FlowCytomix multiplex array according to the manufacturer's instructions.

\section{Calcium flux}

Measurements were taken as described earlier [3]. Briefly, peripheral blood mononuclear cells (PBMCs) were isolated by a standard density gradient centrifugation from $9 \mathrm{~mL}$ of freshly drawn peripheral venous blood and afterward kept in RPMI medium supplemented with $\mathrm{CaCl}_{2}$ (calcium concentration: $2 \mathrm{mM}$ ) throughout the following steps of the procedure. PBMCs were then incubated with the following conjugated anti-human monoclonal antibodies: anti-CD4 PE-Cy7, anti-CD8 APC-Cy7, anti-CXCR3 APC (for the determination of Th1 cells) and anti-CCR4 PE (for the determination of Th2 cells) (all from PharMingen, San Diego, CA, USA) according to the manufacturer's instructions.

Table 1 Clinical characteristics of study participants

\begin{tabular}{llll}
\hline Characteristics & $\begin{array}{l}\text { Healthy donors } \\
n=8\end{array}$ & $\begin{array}{l}\text { RA patients } \\
n=15\end{array}$ & $\begin{array}{l}\text { AS patients } \\
n=10\end{array}$ \\
\hline Age (years) & $50.0 \pm 15.1$ & $53.2 \pm 14.4$ & $52.8 \pm 14.0$ \\
Gender (male/female) & $4 / 4$ & $4 / 11$ & $8 / 2$ \\
Disease duration (years) & - & $13.2 \pm 11.5$ & $15.8 \pm 13.8$ \\
CRP (mg/L) & - & $2.8 \pm 2.1$ & $1.9 \pm 1.5$ \\
Number of patients receiving anti-TNF therapy & - & $15(100 \%)$ & $10(100 \%)$ \\
DAS28 & - & $2.4 \pm 1.0$ & - \\
BASDAI & - & - & $3.2 \pm 2.1$ \\
Number of anti-CCP-negative patients & - & $3(20 \%)$ & - \\
Number of HLA-B27-positive patients & - & - & $8(80 \%)$ \\
\hline
\end{tabular}

Data are expressed as mean \pm SD for continuous variables and as number (percent) for categorical variables 
For monitoring cytoplasmic calcium levels, PBMCs were loaded with calcium-sensitive Fluo-3 and Fura Red dyes according to the manufacturer's recommendations (Invitrogen, Carlsbad, CA, USA). PBMCs were incubated in the presence or absence of $4 \mathrm{nM}$ MGTX or $240 \mathrm{nM}$ TRAM. After recording a baseline for $2 \mathrm{~min}$, cells were activated with $20 \mu \mathrm{g}$ phytohemagglutinin (PHA) and the measurement was continued directly afterward in a kinetic manner for 15 min employing a flow cytometer (Beckman Coulter Gallios $^{\mathrm{TM}}$, Miami, FL, USA). Recordings were evaluated with specific software (FacsKin, Budapest, Hungary) which is based on the calculation of a logistic function for each measurement [4].

\section{Statistics}

Data are expressed as mean \pm SEM. Comparisons were made with unpaired and paired Student's $t$ tests. $p$ values $<0.05$ were considered significant. Statistics were calculated with Microsoft Excel.

\section{Results}

\section{Cytokine levels}

Treatment with MGTX had no significant influence on cytokine production in any of the study groups (Fig. 1a-e). On the contrary, upon treatment with TRAM, the production of IL-1b was decreased in HD samples, while the opposite effect was observed in RA samples (Fig. 1a). The production of IL-5 was increased by treatment with TRAM in RA patients; however, this effect was not observed in
HD and AS (Fig. 1b). Treatment with TRAM decreased the production of TNF-a and IL1-RA by PBMCs in all study groups (Fig. 1c, d). IL-6, IFN-g, IL-10, IL-13, IL-2, IL$12 \mathrm{p} 70$, IL-4 and IL-17A/F levels did not show relevant inhibition with any treatment (Fig. 1e).

\section{Calcium flux}

Figure $2 \mathrm{a}$ shows an example of calcium influx measurements using flow cytometry. In this plot, each dot corresponds to a single cell. AUC values were calculated after fitting a logistic function on each measurement using the FacsKin software. The total amount of calcium influx, represented by AUC values in the histograms, was decreased by MGTX in the Th2 subset of all three study groups (Fig. 2c). On the contrary, TRAM treatment had opposite effects on RA and HD samples: It decreased calcium influx in the Th1 subset of HDs and in Th2 and CD8 cells of RA patients (Fig. 2b-d). Calcium influx was not altered in monocytes in any of the study groups (Fig. 2e).

\section{Discussion}

In this study, we have demonstrated that PBMCs isolated from RA and AS patients react with a different pattern of alterations in cytokine production and calcium influx kinetics compared to HDs. While treatment with MGTX did not influence cytokine production, the application of TRAM resulted in a lower production of TNF-a and IL1RA in all three study groups. Interestingly, inhibition by TRAM had contrary effects on the production of IL-1b and

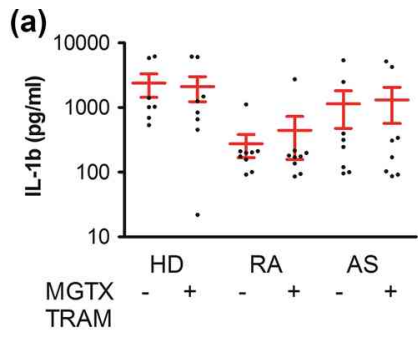

(c)

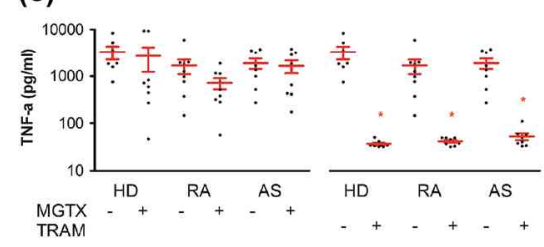

(d)

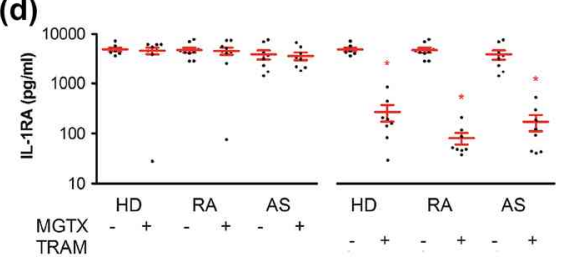

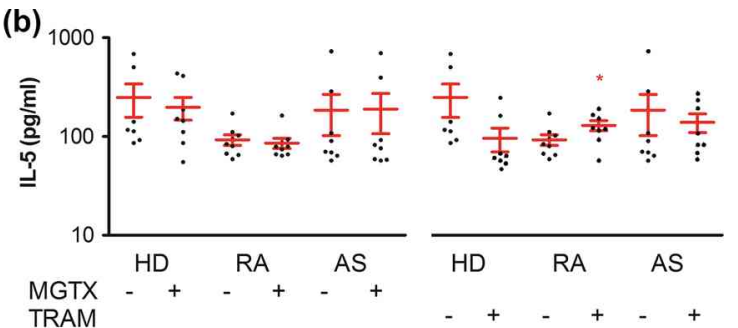

(e)

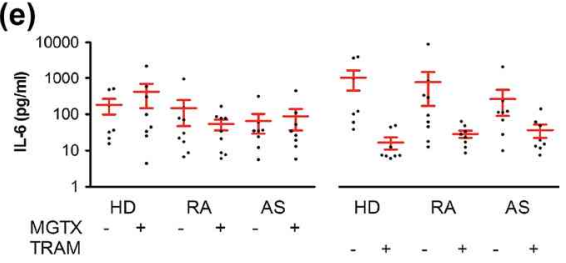

Fig. 1 Concentration of selected cytokines in supernatants of PBMCs isolated from healthy donors (HD), rheumatoid arthritis (RA) and ankylosing spondylitis (AS) patients. PBMCs were cultured over $48 \mathrm{~h}$ in the presence of $160 \mathrm{nM}$ PMA and in the presence or absence of $4 \mathrm{nM}$ margatoxin (MGTX) or $240 \mathrm{nM}$ triarylmethane-34 (TRAM). Data are expressed as mean \pm SEM 
(a)

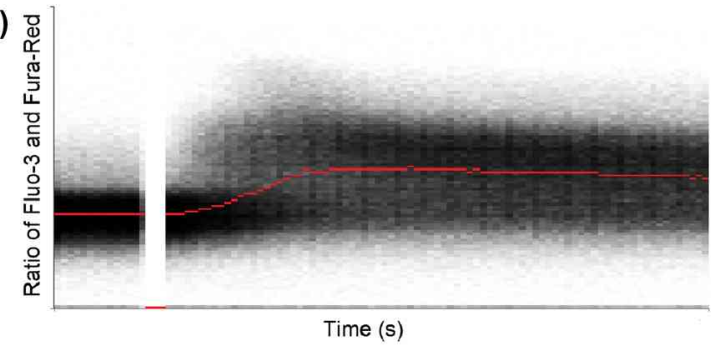

(b)

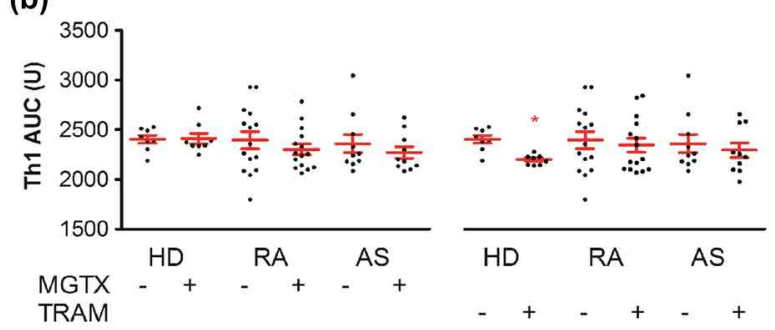

(c)

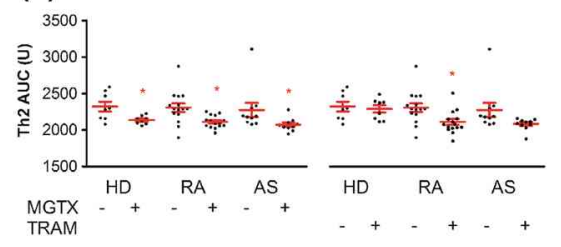

(d)

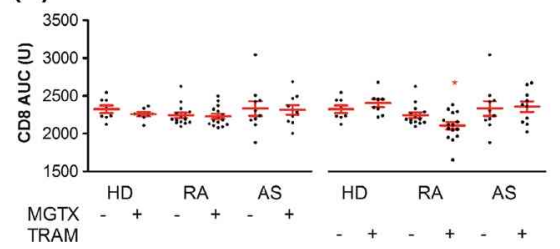

(e)

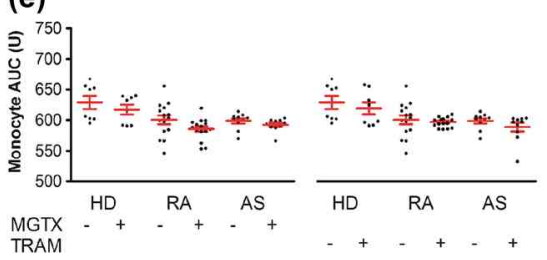

Fig. 2 a Example of calcium influx measurement using flow cytometry. In this plot, each dot corresponds to a single cell. The relative fluorescence ratio of the applied calcium-binding dyes, corresponding to the level of cytoplasmic calcium, is represented on the vertical axis. Median fluorescence values for each time point, represented in red, were used for fitting a logistic function on each

IL-5: While their production was increased by PBMCs of RA patients, this effect was not observed in HD and AS. Likewise, while treatment with MGTX resulted in a similar decrease in calcium influx in the CD4 and Th2 subsets across all study groups, TRAM treatment had opposite effects on RA and HD samples: It decreased calcium influx in the Th2 and CD8 subsets, while only Th1 cells were affected in HDs. The effects of IKCa1 channel inhibition are controversial in samples of RA and AS patients. Although TRAM decreased TNF-a levels in both patient groups, showing a beneficial therapeutic effect, it also decreased IL1-RA levels and increased IL-1b levels in RA, which contributes to a pro-inflammatory response already present in this disease, as observed before [5].

Earlier animal data from Beeton et al. indicated that Kv1.3 inhibitors (SL5 and PAP1) not only suppress calcium signaling and proliferation of autoantigen-specific TEM cells, but also decrease their cytokine production while sparing other classes of T cells. SL5 and PAP1 inhibited IL-2 and IFN-g production by T cells isolated from synovial fluid of a rat model of RA (mainly TEM cells) more effectively than $\mathrm{T}$ cells isolated from peripheral blood of these animals (mainly naïve and TCM cells). However, these Kv1.3 inhibitors were less effective in suppressing the production of TNF-a and IL-4 [2]. Hu et al. [1] also demonstrated the inhibition of IFN-g and IL-5 production by Kv1.3 blockers (MGTX and ShK) in antiCD3/anti-CD28 stimulated freshly isolated human CD4 cells and the inhibition of IFN-g production in TEM cells generated from freshly isolated human CD4 cells. The measurement. b-e Area under the curve (AUC) values of calcium influx kinetics in PBMC subsets isolated from healthy donors (HD), rheumatoid arthritis (RA) and ankylosing spondylitis (AS) patients in the presence or absence of $4 \mathrm{nM}$ margatoxin (MGTX) or $240 \mathrm{nM}$ triarylmethane-34 (TRAM). Data are expressed as mean \pm SEM (Color figure online)

reason for the differences observed in the effects of Kv1.3 channel inhibition between these earlier trials and our results might partly be due to differences in the method of cell stimulation and inhibition. Importantly, we here provide the first data on the effects of $\mathrm{Kv} 1.3$ and IKCa1 inhibitors on human patient samples in RA and AS. While it is not clear how the in vitro observed effects of the applied inhibitors correspond to their immunomodulatory effects in vivo, an advantage of our method over singlecell-based techniques is that physiological cell-to-cell communication between $\mathrm{T}$ cell subsets is maintained in our experimental setup, since individual cell subsets are gated virtually during the evaluation.

A limitation of our study might be the fact that we used different stimuli to measure cytokine production and calcium influx in the presence or absence of potassium channel inhibitors. PMA is a more robust stimulus of cytokine production compared to PHA. Although the main effect of PMA is the activation of protein kinase $C$, it is also known to influence calcium homeostasis [6]. Our supplementary measurements of cytokine production using PHA as a stimulus showed similar but less remarkable tendencies of alterations in cytokine production upon potassium channel inhibition (data not shown). However, this fact does not exclude the possibility that the applied inhibitors might also interact with other cellular signaling mechanisms.

In summary, the application of lymphocyte potassium channel inhibitors has controversial effects on cytokine production and calcium influx patterns in patients with RA 
and AS, questioning its therapeutic relevance. TRAM decreases the secretion of TNF-a and increases the secretion of the Th2 cytokine IL-5 in patients with RA. This would contribute to the shift of the immune balance toward less tissue damage and cellular paucity in RA. Nevertheless, TRAM increases the secretion of $I L-1 b$ and at the same time decreases that of IL1-RA which is unfavorable, since it supports an IL-1b-mediated pro-inflammatory response. Additionally, TRAM also inhibits the short-term activation of Th2 lymphocytes in RA, leaving that of Th1 cells unaffected, probably further contributing to a shift of the inflammatory balance to a pro-inflammatory profile. The differences observed in cytokine response upon inhibition of $\mathrm{Kv} 1.3$ and IKCa1 channels support the differential pathomechanisms of RA and AS.

Acknowledgments This study was supported by an unrestricted research grant from UCB and Hungarian Grant OTKA No. 109451, as well as the Eötvös Fellowship of the State of Hungary. G.T. and A.B. are recipients of the Bolyai Fellowship of the Hungarian Academy of Sciences. This research was supported by the European Union and the State of Hungary, co-financed by the European Social Fund in the framework of TÁMOP 4.2.4. A/2-11-1-2012-0001 'National Excellence Program.' G.T. is an International Society for the Advancement of Cytometry (ISAC) Scholar. L.E.M., G.S. and M.H. were supported by the SFB643 of the German Research Society (DFG) and by the K \& R Wucherpfennig-Stiftung.

\section{Compliance with ethical standards}

Conflict of interest There is no conflict of interest to be declared.

\section{References}

1. Hu L, Pennington M, Jiang Q, et al. Characterization of the functional properties of the voltage-gated potassium channel Kv1.3 in human CD4+ $\mathrm{T}$ lymphocytes. $\mathrm{J}$ Immunol. 2007; 179:4563-70.

2. Beeton $\mathrm{C}$, Wulff $\mathrm{H}$, Standifer NE, et al. Kv1.3 channels are a therapeutic target for T cell-mediated autoimmune diseases. Proc Natl Acad Sci USA. 2006;103:17414-9.

3. Toldi G, Bajnok A, Dobi D, et al. The effects of Kv1.3 and IKCal potassium channel inhibition on calcium influx of human peripheral $\mathbf{T}$ lymphocytes in rheumatoid arthritis. Immunobiology. 2013;218:311-6.

4. Kaposi AS, Veress G, Vásárhelyi B, et al. Cytometry-acquired calcium-flux data analysis in activated lymphocytes. Cytom A. 2008; 73:246-53.

5. Rico MC, Manns JM, Driban JB, et al. Thrombospondin-1 and transforming growth factor beta are pro-inflammatory molecules in rheumatoid arthritis. Transl Res. 2008;152:95-8.

6. Mottola C, Romeo D. Calcium movement and membrane potential changes in the early phase of neutrophil activation by phorbol myristate acetate: a study with ion-selective electrodes. J Cell Biol. 1982;93:129-34. 